\title{
Members of our Early Career Panel highlight key research articles on the theme of antimicrobial resistance
}

\author{
Christopher Alan Brown ${ }^{1} \&$ Sarah J Caswell*,2 \\ ${ }^{1}$ Charles River Laboratories, Chesterford Research Park, Saffron Walden, CB10 1XL, UK \\ ${ }^{2}$ AstraZeneca, Aaron Klug Building, Granta Park, CB21 6GH, UK \\ *Author for correspondence: sarah.caswell@astrazeneca.com
}

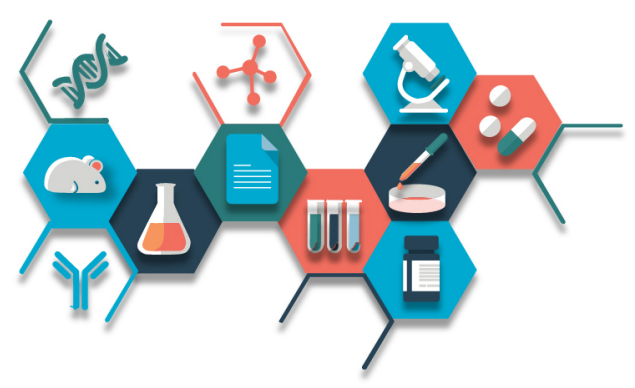

First draft submitted: 8 June 2020; Accepted for publication: 8 June 2020; Published online: 26 June 2020

\section{A $\gamma$-lactam siderophore antibiotic effective against multidrug-resistant gram-negative bacilli}

The increased expression of efflux pumps or lactamases are two mechanisms that can manifest in multidrug resistant (MDR) bacilli which reduce the efficacy of lactam-containing antibiotics. To mitigate these mechanisms, either siderophores can be incorporated into inhibitors to exploit bacterial iron-transport [1] or the $\beta$-lactam pharmacophore can be modified to reduce hydrolysis by lactamases. This article combines both approaches with YU253434 [2], a novel $\gamma$-lactam pyrazolidinone pharmacophore appended by a catechol-phthalimide siderophore [3]. Minimum inhibitory concentration (MIC) studies of YU253434 against MDR clinical isolates of Pseudomonas aeruginosa, Klebsiella pneumoniae and Escherichia coli compare favorably against contemporary clinical $\beta$-lactam therapeutics, including ceftazidime and carbapenem. Uptake of YU253434 via iron-transports was supported by the observation that increased iron concentrations negatively affected MICs in a manner similar to the siderophore cefiderocol. Stability studies of YU253434 showed significant resistance to hydrolysis against four classes of $\beta$-lactamases and drug-like properties are comparable with conventional intravenous-dosed $\beta$-lactam antibiotics. Target elucidation studies showed that the transpeptidase PBP3 enzyme showed an inhibitory doseresponse with YU253434 in conjunction with a positive shift in thermal melt temperature of PBP3 and YU253434 (DSF assay). A crystal structure of YU253434 bound to PBP3 was also resolved (PDBid 6VOT).

- Written by CA Brown

\section{Structure \& molecular recognition mechanism of IMP-13 metallo- $\beta$-lactamase}

$\beta$-lactam antibiotics contain a $\beta$-lactam ring in their molecular structure and are the most commonly prescribed antibiotics for both gram-positive and gram-negative bacterial infections. One primary bacterial resistance mechanism which has arisen with the use of these antibiotics is the production of $\beta$-lactamase enzymes that hydrolyze and inactivate $\beta$-lactam ring-containing antibiotics. $\beta$-Lactamase enzymes include metallo- $\beta$-lactamases which can hydrolyze all bicyclic $\beta$-lactams and may be propagated through horizontal gene transfer. Imipenemases (IMPs) represent one of the major groups of class B1 metallo- $\beta$-lactamases found in Gram-negative pathogens and to date, no clinically available inhibitors have been identified for class B metallo- $\beta$-lactamases. Here [4], the authors use a combined structural approach of $\mathrm{x}$-ray crystallography, solution NMR spectroscopy and molecular dynamics to reveal novel high-resolution structural and mechanistic information of IMP-13 metallo- $\beta$-lactamase. Comparisons between apo- and IMP-13 complexed with four clinically relevant carbapenem antibiotics revealed differential dynamics of the active site loop in open and closed conformations which ultimately determines active site accessibility. This exciting structural data gives an insight into the mechanism of antibiotic binding, how the L1-loop contributes to the mechanism of cleavage and the specific regions of IMP-13 which could therefore be targeted for small molecule inhibition in drug discovery.

- Written by SJ Caswell 
First detection of autochthonous extensively drug-resistant NDM-1 P. aeruginosa ST235 from a patient with bloodstream infection in Italy, October 2019

This study [5] highlights the importance of continually investigating bacterial genomes to understand mechanisms and development of antibiotic resistance using whole genome sequencing. This information will help to understand the epidemiology of infection and contribute to minimizing the spread of antimicrobial resistant bacterial strains. A combination of microbial identification and antibiotic susceptibility testing using the VITEK2 system (bioMérieux, Marcy-l'Étoile, France) plus whole genome sequencing, allowed the identification of the first known infection by the New Delhi metallo- $\beta$-lactamase (NDM)-producing P. aeruginosa ST235 strain in Italy. The ST235 strain has multidrug and extensive-drug resistance (MDR/XDR) and is associated with high mortality rates. Bioinformatic analyses identified a number of antibiotic resistance markers, including NDM-1, multiple $\beta$-lactamases, multidrug efflux pump complexes (which reduce the cellular antibiotic concentration), the virulence gene ExoU and closely related strains isolated in Serbia and Italy. Due to understanding the threat posed by this strain, in-depth epidemiological analyses are being pursued to investigate the origin and spread of this isolate and infection control measures have been implemented at the elderly healthcare and rehabilitation facility in which this infection was first identified.

- Written by SJ Caswell

Financial \& competing interests disclosure

CA Brown is an employee of Charles River Laboratories inc. SJ Caswell is an employee of AstraZeneca. The authors have no other relevant affiliations or financial involvement with any organization or entity with a financial interest in or financial conflict with the subject matter or materials discussed in the manuscript apart from those disclosed.

No writing assistance was utilized in the production of this manuscript.

Open access

This work is licensed under the Attribution-NonCommercial-NoDerivatives 4.0 Unported License. To view a copy of this license, visit http://creativecommons.org/licenses/by-nc-nd/4.0/

\section{Referecnes}

1. Aoki T, Yoshizawa H, Yamawaki K et al. Cefiderocol (S-649266), A new siderophore cephalosporin exhibiting potent activities against Pseudomonas aeruginosa and other gram-negative pathogens including multi-drug resistant bacteria: structure activity relationship. Eur. J. Med. Chem. 155, 847-868 (2018).

2. Goldberg J, Nguyen $\mathrm{H}, \mathrm{Kumar} \mathrm{V}$ et al. A $\gamma$-lactam siderophore antibiotic effective against multidrug-resistant gram-negative bacilli. J. Med. Chem. 63(11), 5990-6002 (2020).

3. Starr J, Brown MF, Aschenbrenner L et al. Siderophore receptor-mediated uptake of lactivicin analogues in gram-negative bacteria. $J$. Med. Chem. 57(9), 3845-3855 (2014).

4. Softley CA, Zak KM, Bostock MJ et al. Structure and molecular recognition mechanism of IMP-13 metallo- $\beta$-lactamase. Antimicrob. Agents Chemother. 64(6), e00123-20 (2020).

5. Loconsole D, Accogli M, Monaco M et al. First detection of autochthonous extensively drug-resistant NDM-1 Pseudomonas aeruginosa ST235 from a patient with bloodstream infection in Italy, October 2019. Antimicrob. Resist. Infect. Control 9, 73 (2020). 